\title{
Performance analysis of $\kappa-\mu$ distribution for Global Positioning System (GPS) L1 frequency-related ionospheric fading channels
}

\author{
Alison Moraes ${ }^{1, *}$, Jonas Sousasantos ${ }^{2}$, Eurico Rodrigues de Paula ${ }^{3}$, Josué Jürgen Popov Pereira da Cunha ${ }^{4}$, \\ Vicente Carvalho Lima Filho ${ }^{2}$, and Bruno Cesar Vani ${ }^{5}$ \\ ${ }^{1}$ Instituto de Aeronáutica e Espaço - IAE, São José dos Campos, SP 12228-904, Brazil \\ 2 Instituto Tecnológico de Aeronáutica - ITA, São José dos Campos, SP 12228-900, Brazil \\ ${ }^{3}$ Instituto Nacional de Pesquisas Espaciais - INPE, São José dos Campos, SP 12227-010, Brazil \\ ${ }^{4}$ McKinsey \& Company, São Paulo, SP 04717-004, Brazil \\ ${ }^{5}$ Instituto Federal de Educação, Ciência e Tecnologia de São Paulo - (IFSP-PEP), Presidente Epitácio, SP 19470-000, Brazil
}

Received 15 June 2018 / Accepted 20 March 2019

\begin{abstract}
The present work aims to evaluate the application of the $\kappa-\mu$ distribution as a representation of the fading effect caused by the phenomenon of scintillation on $L$-band transionospheric radio links. The ionospheric scintillation is a phenomenon defined as a rapid variation in the amplitude and phase of electromagnetic wave signals. This phenomenon starts in the first hours of the night, at latitudes near the geomagnetic equator. Scintillation occurs when radio signals cross ionospheric irregularities, also known as plasma bubbles. These plasma bubble structures are generated after the sunset due to instabilities in the $F$ region of ionosphere. Distributions with non-single parameter usually present better results, however, this point requires further investigation by comparing different models. The goals of this study are: (1) the modeling of experimental data using the $\kappa-\mu$ distribution; (2) the $\kappa-\mu$ parameters characterization for empirical data and the evaluation of parameters estimation based in different approaches; (3) the comparison between the distribution proposed and other models adopted in the literature in order to verify the performance of two parameter models. The results of the analysis performed showed that the $\kappa-\mu$ distribution presents good fitting of the empirical scintillation data. These fitting results were calculated through the chi-square fit test under which the values reveal fair $E\left[\chi^{2}\right]$ for $\kappa-\mu$ distribution in most of the cases. The evaluation of $\kappa-\mu$ parameters suggests that the distribution has a more conservative outcome than in the distributions traditionally used, but being a legitimate approximation due to its adjustable features in the tail region of the distribution. Typical pairs of $\kappa-\mu$ coefficients are presented for theoretical works. The comparison of $\kappa-\mu$ distribution to Rice, Nakagami- $m$ and $\alpha-\mu$ models showed that $\kappa-\mu$ is capable of describing more severe scintillation scenarios where the tail of the distribution is more raised in comparison to the other models.
\end{abstract}

Keywords: ionospheric scintillation / fading distributions / statistical modeling / GNSS availability / GNSS positioning issues

\section{Introduction}

In Global Positioning System (GPS) L1 (1575.42 MHz) single-frequency receivers, such as those used in aviation, the ionosphere is one of the largest sources of error in positioning (Klobuchar, 1987). In this way, much of the efforts and research carried out in this area have the objective of understanding these errors, modeling them and developing strategies for mitigation. As a complicating factor of the usage of GPS-L1 for navigation,

*Corresponding author: aom@ita.br a plethora of phenomena occur in the ionosphere over the Brazilian territory, making these errors even worse and potentially dangerous. The high declination of the geomagnetic field strongly influences the ionospheric phenomenology. The Equatorial Ionization Anomaly (EIA), the South Atlantic Magnetic Anomaly (SAMA), and the generation of plasma bubbles during the night time period are examples of such phenomenology. These are peculiarities that make this region unique and its problems for operation with GPS much more worrisome. Figure 1 illustrates a pictorial representation of some of these characteristics. The color bar indicates the geomagnetic total 


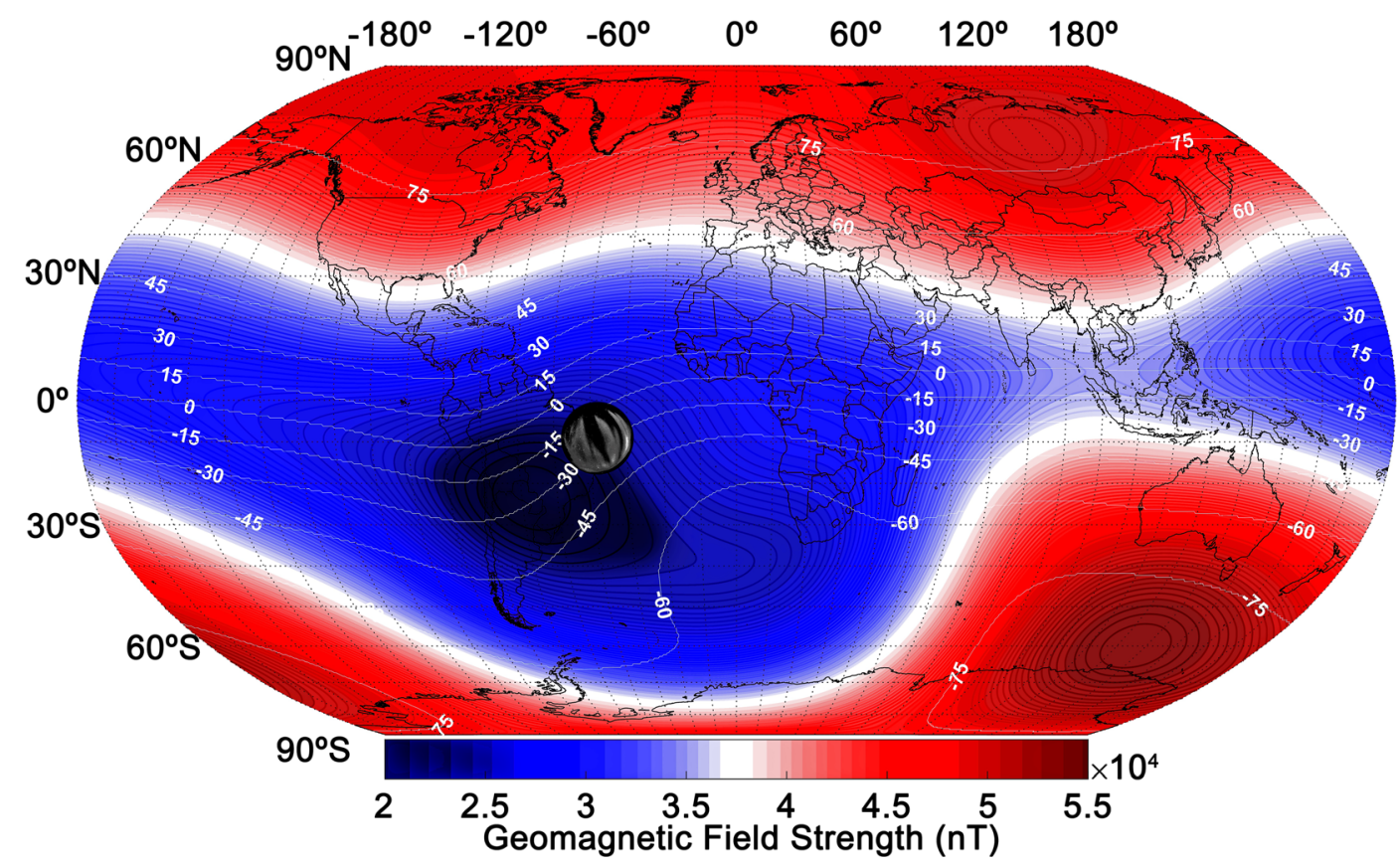

Fig. 1. Some of the phenomena which take place in the Brazilian region and affect the GPS performance. SAMA (darker blue region), and plasma bubbles (black striation in the circle).

field intensity at altitude of $350 \mathrm{~km}$ with colder (hotter) colors indicating lesser (larger) values. In the South American/ Brazilian region a deep decrease in the intensity is present as showed by the darker blue contours, this region is the SAMA. Also, the figure presents, in white lines, the inclination component of the geomagnetic field. As may be noticed, over the Brazilian region the difference between the geomagnetic and geographic axes is the larger one. Both information are based on the 12th generation of the International Geomagnetic Field Reference Field, IGRF12 (Thébault et al., 2015) from which values were obtained to produce the figure. Additionally, a registry of equatorial plasma bubbles obtained through a charged coupled device imager using a filter which captures only emission in the wavelength of the atomic oxygen (6300 $\AA$ ) is superposed over the figure to illustrate the spatial domain of such structures. The darker regions correspond to strong depletions in the ionospheric plasma density and transionospheric radio signals crossing these regions may suffer severe scintillation.

The propagation delay caused by the ionosphere is a great source of errors for single frequency GPS positioning. In order to mitigate these effects for aviation user, augmentation systems were developed with the goal of improving navigation accuracy, integrity and availability. In Datta-Barua et al. (2010), for example, it was proposed a threat model capable to deal with those ionospheric gradients which may affect augmentation systems. It is important to note that this ionospheric threat parameterization of Datta-Barua et al. (2010) was compatible to the ionosphere at North America region. However, adaptations in this model are necessary for operation in the Brazilian territory, so the augmentation systems may be prepared to deal with the great majority of the cases involving high dynamic gradients in the ionosphere.
Another problem caused by ionosphere is the oscillation caused by the plasma bubbles, called ionospheric scintillation. Their effects imply possible availability issues, which cause the dilution of precision, consequently decreasing the positioning performance (Moraes et al., 2017; Marques et al., 2018). Because of this, limitations on the navigation performance can even lead to eventual problems in the landing operation or, in extreme cases, to the impossibility of this operation by using satellite navigation. The aforementioned is a concern in the use of augmentation systems at night in regions of low latitudes and, therefore, characterizing and modeling the scintillation is essential to evaluate the use of augmentation systems under these conditions.

In this work, a statistical analysis of the ionospheric scintillation is performed for GPS users under low latitude and high solar flux conditions. Statistical distributions such as Rice, Nakagami- $m$ and $\alpha-\mu$ have been used in the efforts to better characterize these phenomena (Fremouw et al., 1978, 1980; Humphreys et al., 2009; Moraes et al., 2012; Guo et al., 2017). The results suggested that two parameter distributions could represent the empirical data more precisely due to the additional Degree Of Freedom (DOF) (Moraes et al., 2012). Notwithstanding, this proposition remain unverified, thus the comparison of these models with other two parameter distribution is needed. The $\kappa-\mu$ distribution was chosen because it is advisable for line-of-sight applications (Yacoub, 2007b) such as in case of transionospheric links. The performance of the $\kappa-\mu$ model will be evaluated to statistically characterize the scintillation and compared to that of models traditionally used in the literature. The discussions will also show the particularities and benefits of each of the models, which can help in the task of modeling this phenomenon. This kind of distribution may be used to estimate the Global Navigation 
Satellite System (GNSS) receiver errors on the Phase Locked Loop (PLL) and support for example models like in Veettil et al. (2018).

In Section 2, details about the database used and how the measurements were processed are presented. Section 3 formally presents all models that are used in the literature to describe the statistics of ionospheric scintillation, and Section 4 introduces the $\kappa-\mu$ model. In Section 5 a comparison of the models, in the fitting of the empirical data, is performed. Finally, the Section 6 summarizes the findings of the paper.

\section{Scintillation measurements and dataset}

The experimental data used in this work was acquired between December 14, 2001 and January 14, 2002, a period that embraces the equatorial spread $F$ season in Brazil (Abdu et al., 1992; Sobral et al., 2002) and contains large number of scintillation events in all ranges of amplitude scintillation strength. These months belong to the period of the peak of the solar cycle 23 and the average F10.7 cm solar flux was $176\left(10^{-22} \mathrm{Wm}^{-2} \mathrm{~Hz}^{-1}\right)$. The GPS scintillation monitor receiver was installed in Brazil, in the city of São José dos Campos $\left(23.1^{\circ} \mathrm{S} ; 45.8^{\circ} \mathrm{W}\right)$, with a geomagnetic dip latitude of $17.3^{\circ} \mathrm{S}$, in the southern crest of EIA. Stolle et al. (2006) showed that this period presents the largest occurrence rate of spread $F$ over the Brazilian longitudinal sector (Figure 6 in their paper). In fact this region presented the most prominent occurrence rate of spread $F$ within their dataset, thus suggesting strong scintillation conditions, suitable for the investigation proposed here.

Scintillation observations were recorded every night, starting at 18:00 LT and lasting until the next day at 06:00 LT. Normally, scintillation is observed between sunset and local midnight, but post-midnight cases may happen (Sousasantos et al., 2018). For this analysis, only scintillation data from GPS satellites with elevation greater or equal than $30^{\circ}$ were considered. The amount of records acquired during this campaign with noticeable scintillation corresponds to approximately $60 \mathrm{~h}$ of GPS data, for more details about the dataset see Moraes et al. (2012). This data was acquired by a scintillation monitor receiver developed at Cornell University called SCINTMON. SCINTMON operates only for L1 band (1575.42 MHz) and it has 11-channels for GPS reception at 50 samples-per-second and one dedicated for noise floor estimation (Beach \& Kintner, 2001).

Figure 2a shows an example of a GPS signal recorded by SCINTIMON and affected by scintillation. It is possible to observe two cases, in blue, which show that the received signal is under the interference of ionospheric scintillation. Each of those blue periods is a bubble crossing the GPS signal propagation path. Analyzing in detail Figure 2a, it is observed that the first occurrence of ionospheric bubbles lasts about $45 \mathrm{~min}$, between 23:25 and 00:10 UT. After a 30-minute interval, another period of scintillation occurs, now lasting $55 \mathrm{~min}$, but now less intense than the first one. In the first bubble period, there was a fading event that goes below $20 \mathrm{~dB}-\mathrm{Hz}$, which is considered to be below the operating threshold of many receivers (Seo et al., 2011). Cases like that make the link to the satellite to be momentarily unavailable, affecting positioning. These registered cases frequently result in loss of lock events,
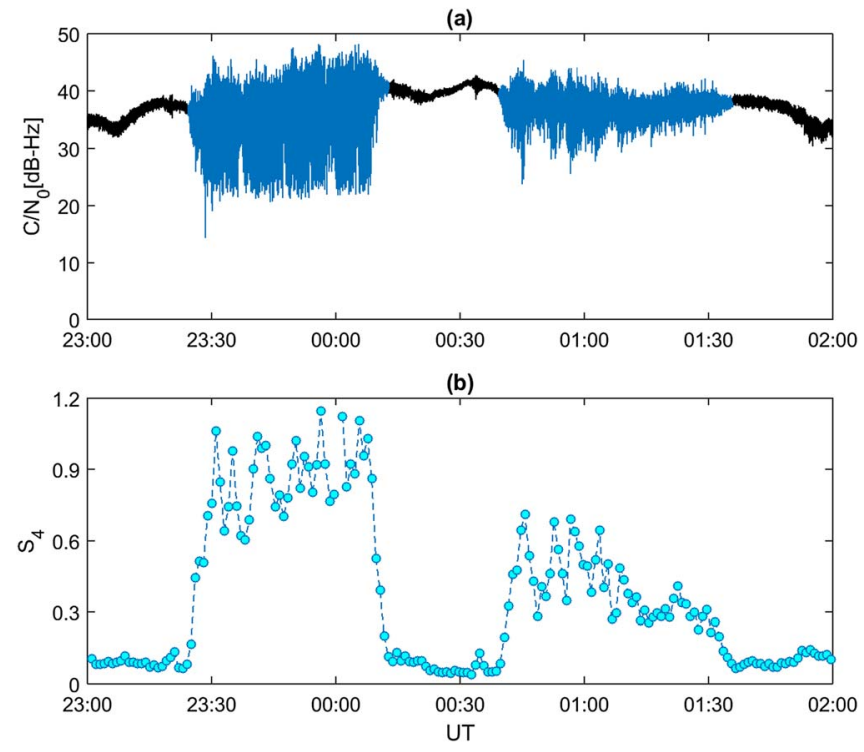

Fig. 2. GPS example of received signal for PRN 28 in the night between 14 and 15 of December of 2001. (a) GPS signal affected by ionospheric bubbles in blue. (b) The respective $S_{4}$ index computed.

overall over the region of ionization anomaly crests as discussed in Xiong et al. (2016).

The index that quantifies the strength of amplitude scintillation is called the $S_{4}$ index, given by Yeh \& Liu (1982):

$$
S_{4}=\sqrt{\frac{\left\langle I^{2}\right\rangle-\langle I\rangle^{2}}{\langle I\rangle^{2}}}
$$

where $I=R^{2}$ is the intensity, $R$ is the amplitude envelope of the received signal and the $\langle>$ corresponds to the ensemble average that, in practice, is computed every $60 \mathrm{sec}$ (i.e., 3000 samples). Figure $2 \mathrm{~b}$ shows the respective $S_{4}$ index for the received signal from Figure $2 \mathrm{a}$. To show in more detail as the $S_{4}$ index varies the strength of scintillation, Figure 3 provides three examples of $1 \mathrm{~min}$ data recorded, with different levels of scintillation. These cases illustrate how the severity of the fading events enhances as $S_{4}$ increases. This figure provides a notion of how complicated it is for the receiver to remain in lock as the signal quality deteriorates. Therefore, characterizing the statistics of these fades is relevant to estimate the effects of this phenomenon and also to support the development of signal processing techniques and positioning algorithms that will minimize these effects in the receiver and consequently in navigation (see for example Marques et al., 2018).

\section{Amplitude scintillation distributions}

In the late 1970 s and early 1980 s, the studies presented by Fremouw et al. $(1978,1980)$ intended to evaluate statistical models that best described the ionospheric scintillation under low latitudes. At that time, and for the dataset available, the authors concluded that the Nakagami- $m$ distribution presented the best fit performance. Later, Banerjee et al. (1992) presented 

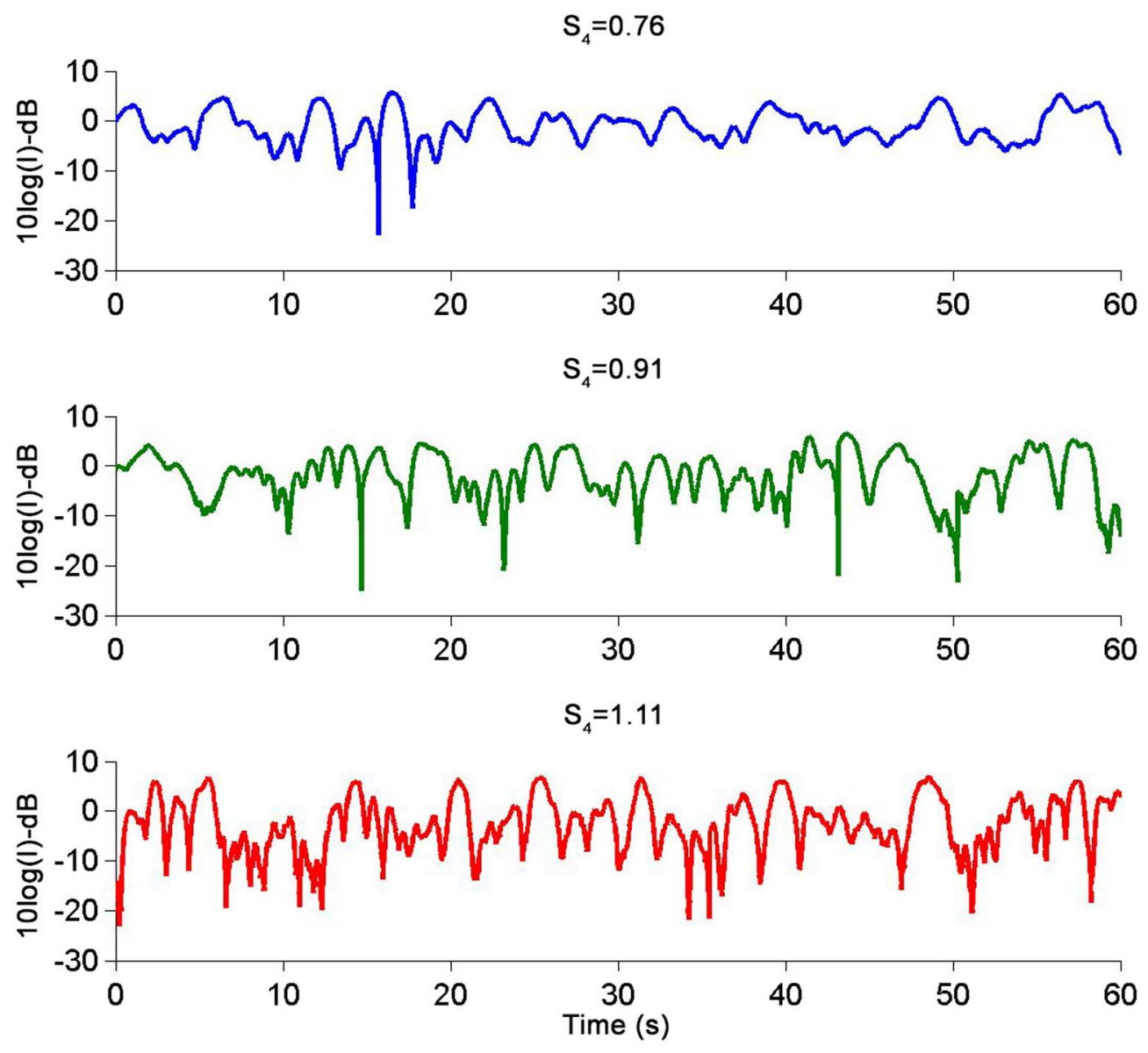

Fig. 3. Three examples of scintillation data showing how the signal quality degrades according to the $S_{4}$ increasing.

results confirming these conclusions. Almost two decades later, in Humphreys et al. (2009) the authors performed similar analysis. Their conclusion was that Rice distribution was the most appropriated for fitting experimental data. Subsequently, an investigation by Moraes et al. (2012) showed the advantage of using a two-parameter model, with the proposed use of the $\alpha-\mu$ distribution (Yacoub, 2007a). Their results showed that the additional DOF from this distribution resulted in a superior fit performance. In this section, the Nakagami- $m$, Rice and $\alpha-\mu$ models will be presented, assuming for those cases that the received signal is normalized so that, the expected value of receiver intensity is $1\left(E\left[R^{2}\right]=1\right)$.

\subsection{Nakagami-m Model}

The probability distribution function of Nakagami- $m$ model is as given by Simon \& Alouini (2006):

$$
f_{R}(r)=\frac{2 m^{m} r^{2 m-1}}{\Gamma(m)} e^{-m r^{2}}
$$

where $r \geq 0$ is the amplitude value of the scintillation envelope $R, \bar{\Gamma}(\ldots)$ is the Gamma function. The $m$ parameter of equation (2) is relation of moments given by:

$$
m=\frac{E^{2}\left[R^{2}\right]}{\left\{E\left[R^{4}\right]-E^{2}\left[R^{2}\right]\right\}}
$$

The parameter $m$ of Nakagami- $m$ model follows the relation $m=1 / S_{4}^{2}$ in relation to the scintillation index $S_{4}$. Figure 4 a plots $f_{R}(r)$ versus $r$ for different values of $S_{4}$, using this relation.

\subsection{Rice model}

The Rice probability distribution function is given by Simon \& Alouini (2006):

$$
f_{R}(r)=2 r(1+K) I_{0}\left(2 r \sqrt{K+K^{2}}\right) e^{-K-r^{2}(1+K)}
$$

where, $I_{0}$ is the modified Bessel function of the first kind and order zero, and $K$ variable is the Rician parameter. There is a relationship of the Rician parameter $K$ with the $m$ parameter from Nakagami- $m$ model. This relation is given by:

$$
K=\frac{\sqrt{m^{2}-m}}{m-\sqrt{m^{2}-m}}
$$

Based on the relationship $m=1 / S_{4}{ }^{2}$ it is possible to relate $S_{4}$ with the $K$ parameter as well. Figure 4 b illustrates Rice density function of equation (4) for different values of $S_{4}$.

\section{$3.3 \alpha-\mu$ model}

Yacoub (2007a) proposed a general fading model that considers the received signal as a composition of multipath waves 

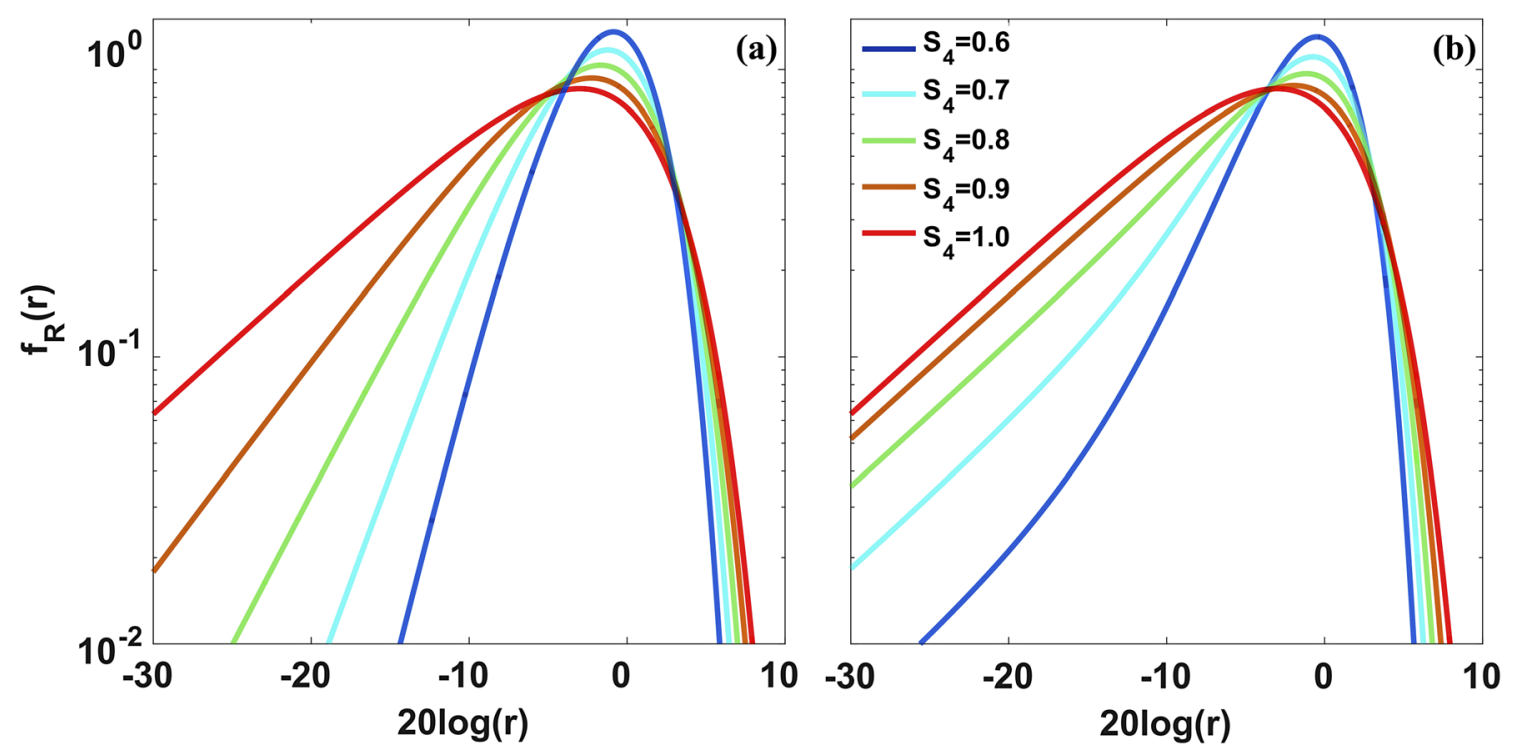

Fig. 4. Five shapes of density function for different values of $S_{4}$ for Nakagami- $m$ model (a), and Rice model (b).

from a non-homogeneous medium. This model is parameterized by the coefficients $\alpha$ and $\mu$, where $\alpha$ represents the modulus of the sum of the multipath components and $\mu$ is the number of multipath components. The $\alpha-\mu$ probability density function for $E\left[R^{2}\right]=1$ is given by Moraes et al. (2012):

$$
f_{R}(r)=\frac{\alpha r^{\alpha \mu-1}}{\xi^{\alpha \mu / 2} \Gamma(\mu)} \exp \left(-\frac{r^{\alpha}}{\xi^{\alpha / 2}}\right)
$$

where $\xi=\frac{\Gamma(\mu)}{\Gamma(\mu+2 / \alpha)}$.

Unlike the Nakagami- $m$ and Rice models, where the $S_{4}$ index is directly related to the parameter $m$ or $K$, the $\alpha-\mu$ model has a different equality of moments for its parameters, given by Yacoub (2007a):

$$
\frac{E^{2}\left[R^{\beta}\right]}{E\left[R^{2 \beta}\right]-E^{2}\left[R^{\beta}\right]}=\frac{\Gamma^{2}(\mu+\beta / \alpha)}{\Gamma(\mu) \Gamma(\mu+2 \beta / \alpha)-\Gamma^{2}(\mu+\beta / \alpha)}
$$

Based on the equation (7), the $\alpha-\mu$ parameters can be estimated by the field data. For example, as there are two unknowns, assuming $\beta=1$ and $\beta=2$, in the left side of equation (7) there will be two equations and the pair $\alpha-\mu$ may be found.

Assuming $\beta=2$ in equation (7), it is possible to establish a relationship of $\alpha-\mu$ coefficients with equation (3) and consequently $S_{4}$, this relation is given by Moraes et al. (2012):

$$
S_{4}^{2}=\frac{\Gamma(\mu) \Gamma(\mu+4 / \alpha)-\Gamma^{2}(\mu+2 / \alpha)}{\Gamma^{2}(\mu+2 / \alpha)}
$$

Analyzing equation (8) it is possible to observe that there will be infinite pairs of $\alpha-\mu$ coefficients that satisfy this equation for a single value of $S_{4}$. Indeed this is the advantage of this model in comparison to the Nakagami- $m$ and Rice models, since the additional DOF allows a fine topological adjustment of model according to the empirical data, in particular in the tail of the distribution. The estimation of the $\alpha-\mu$ coefficients was discussed in details in Moraes et al. (2012, 2014). Figure 5 plots various shapes of $\alpha-\mu$ probability density function for different values of $S_{4}$. It worth observe that for the same $S_{4}$ value, as $\alpha$ increases the tail of the distribution rises, which means that the received signal will present more fading events as discussed in Moraes et al. (2014, 2018a, 2018b).

In Moraes et al. $(2012,2014)$, they show that the estimation of $\alpha$ and $\mu$ pairs based on the equality of moments of equation (7) provides very good results in describing the distribution of amplitudes scintillation data. Finally, according to Yacoub (2007a) this model becomes the Nakagami- $m$ distribution in the particular case where $\alpha=2$ and $\mu=m$.

\section{The $\kappa-\mu$ model}

The $\kappa-\mu$ model was proposed in Yacoub (2007b), and it is based on the same assumption of $\alpha-\mu$ model, namely, the received signal is composed of cluster of multipath waves that propagates in a non-homogeneous channel. According to Yacoub (2007b), the probability density function of $\kappa-\mu$ distribution for the normalized envelope is given by:

$$
\begin{aligned}
f_{R}(r)= & \frac{2 \mu(1+\kappa)^{\left(\frac{\mu+1}{2}\right)}}{\kappa^{\left(\frac{\mu-1}{2}\right)} \exp (\kappa \mu)} r^{\mu} \\
& \times \exp \left[-\mu(1+\kappa) r^{2}\right] I_{\mu-1}[2 \mu \sqrt{\kappa(1+\kappa)} r]
\end{aligned}
$$

where $I_{\mu-1}$ is the modified Bessel function of the first kind and order $\mu-1, \kappa>0$ represents the ratio between the dominant components versus the power of the scattered wave, and $\mu$ is given by:

$$
\mu=\frac{E^{2}\left[R^{2}\right]}{V\left[R^{2}\right]} \frac{1+2 \kappa}{(1+\kappa)^{2}}
$$

such that $V$ denotes the variance. Analyzing equation (10) it is possible to observe that $\mu$ can be easily computed with field 

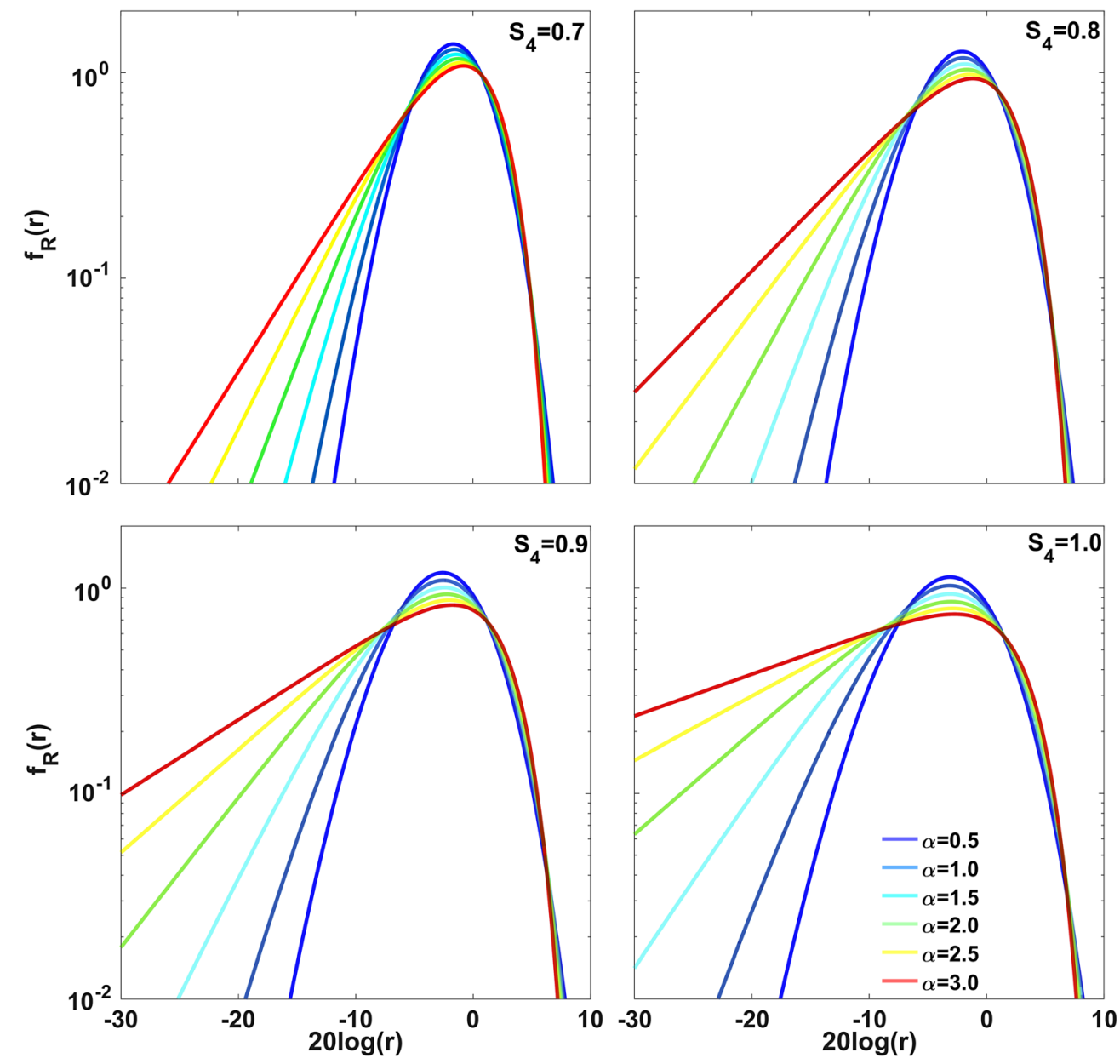

Fig. 5. Various shapes of $\alpha-\mu$ density function for single values of $S_{4}$ showing the versatility of this model.

data if $\kappa$ is available. In Yacoub (2007b), it is shown that $\kappa$ may be calculated through the following expression:

$$
\kappa=\left(\frac{\sqrt{2}\left[E\left(R^{4}\right)-1\right]}{\sqrt{2 E^{2}\left(R^{4}\right)-E\left(R^{4}\right)-E\left(R^{6}\right)}}-2\right)
$$

Similarly to the $\alpha-\mu$ model, it is convenient to have a relationship between the $\kappa-\mu$ distribution and the $S_{4}$ index. This relation is expressed by:

$$
S_{4}=\sqrt{\frac{1+2 \kappa}{\mu\left(1+\kappa^{2}\right)}}
$$

Ideally, the pair may be found by computing firstly equation (11) and then equation (10), but again, like the $\alpha-\mu$ model, there will be infinite pairs of $\kappa-\mu$ that satisfies equation (12) for a single value of $S_{4}$. Figure 6 illustrates how $\kappa$ and $\mu$ varies according to different $S_{4}$ values showing that, usually, $\kappa$ decreases as $\mu$ increases. Figure 7 shows different shapes of the probability density function, $f_{R}(r)$, varying as a function of $S_{4}$. It is worth to notice that, for the same $S_{4}$, the tail of the

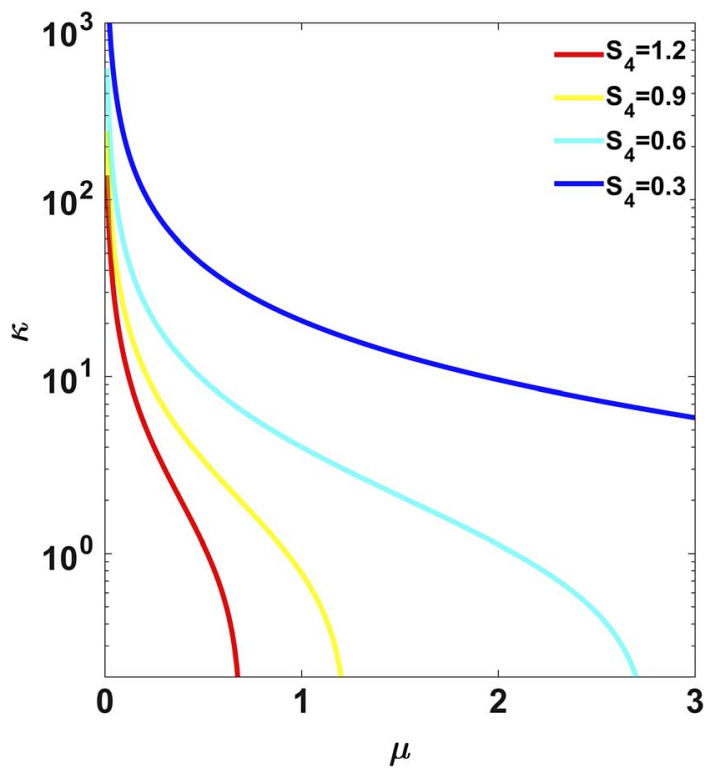

Fig. 6. Relationship of fading coefficients $\kappa-\mu$ for distinct values of $S_{4}$. As the curves describes, $\kappa$ decreases as $\mu$ increases. 

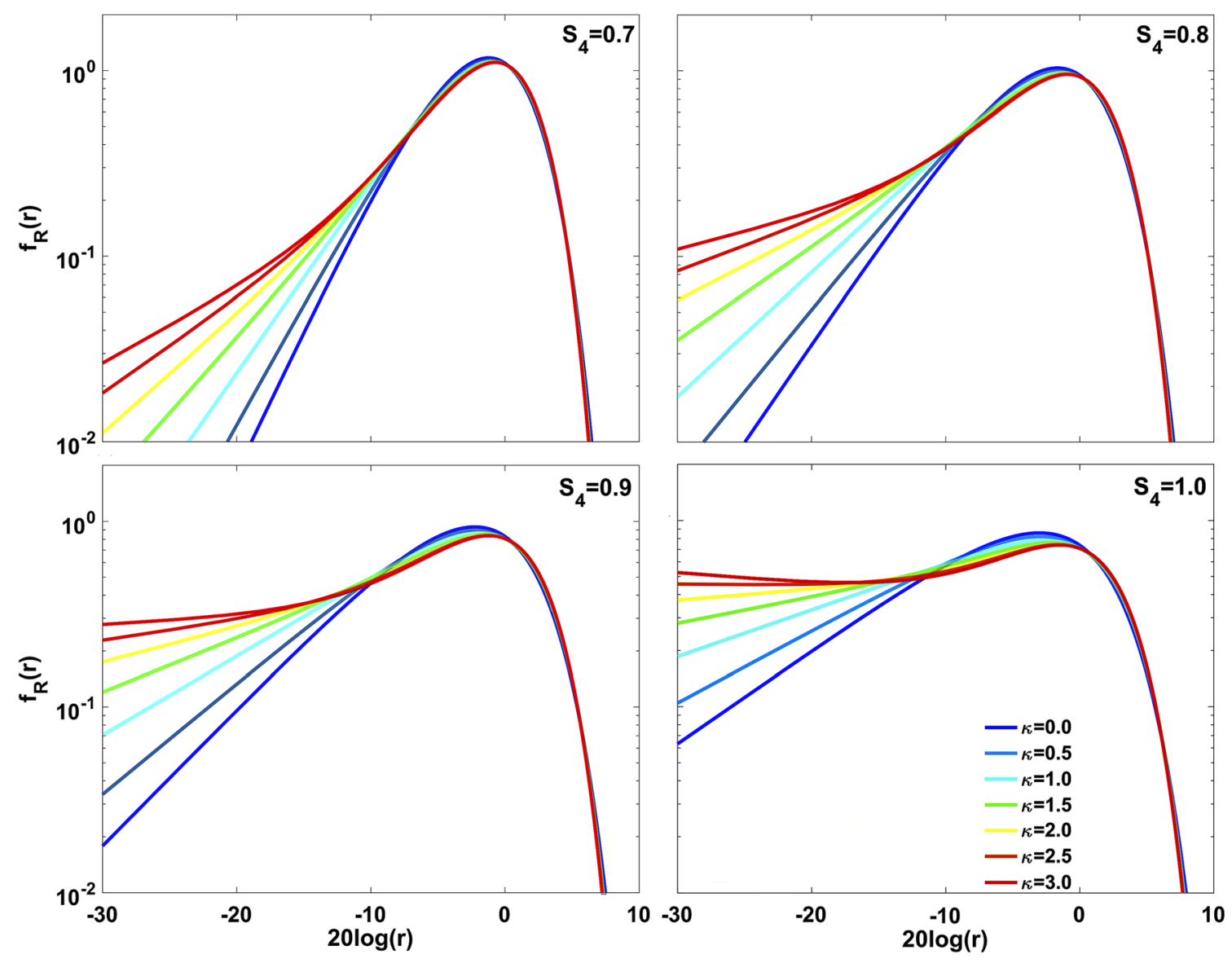

Fig. 7. Various shapes of $\kappa-\mu$ density function for different values of $S_{4}$.

distribution tends to rise as $\kappa$ increases. For example, comparing the curve for $S_{4}=1.0$ from Figure 7 to the respective one presented in Figure 5 it is possible to note that the $\kappa-\mu$ model has a relatively raised tail. Therefore, it is reasonable to argue that this model presents a more conservative estimation, corresponding to a statistical description of a greater occurrence of fades.

\subsection{Evaluation of $\mu-\kappa$ coefficients estimation}

Equation (12) established a relationship between the $S_{4}$ index and the fading coefficients $\kappa-\mu$. In order to estimate the pair of coefficients $\kappa$ and $\mu$ it is necessary to solve equations (10) and (11), respectively. In practice, when performing this procedure, it was noticed that the estimation of $\kappa$ through equation (11) failed. A term-by-term analysis in these failure cases suggests that the $R^{6}$ term in the denominator of the equation (11) is responsible for the existence of such values in the final calculation, once it may imply in a negative term in the square root of the denominator. Table 1 shows the total number of cases available as function of $S_{4}$ and also the percentage of these cases when the estimator was able to estimate $\kappa$. It is possible to observe that the successful cases increase according to the $S_{4}$ increasing. Based on the percentage of successful cases it is possible to conclude that this estimator is not effective for evaluation of datasets like this, containing 3000 samples. Table 1 also shows the average values of $\kappa$ for the estimated cases.
Table 1. Total of cases available and the percentage of cases where $\kappa$ was successfully estimated.

\begin{tabular}{cccc}
\hline$S_{4}$ & Cases (min) & Successful $(\%)$ & $E[\kappa]$ \\
\hline 0.4 & 742 & 2.2 & 5.4 \\
0.5 & 526 & 7.3 & 121.11 \\
0.6 & 338 & 16.3 & 8.69 \\
0.7 & 243 & 24.8 & 4.38 \\
0.8 & 161 & 34.5 & 5.84 \\
0.9 & 111 & 49.1 & 5.96 \\
1.0 & 44 & 53.5 & 1.73 \\
\hline
\end{tabular}

Based on the limitations of the moment-based estimator of the $\kappa-\mu$ distribution, an alternative approach was adopted to estimate the fading coefficients. It was chosen a weighted least square fit approach based on Coleman \& Li (1994, 1996), such that the interior-reflective Newton method adopted assures quadratically global convergence. The procedure is capable to solve problems of nonlinear last-squares curve fitting in the following form:

$$
\min _{x}\|f(x)\|_{2}^{2}=\min _{x}\left[f_{1}(x)^{2}+f_{2}(x)^{2}+\ldots+f_{n}(x)^{2}\right] \text {, where }
$$
$f: \mathfrak{R}^{\mathrm{n}} \rightarrow \mathfrak{R}^{1}, l w \in\{\mathfrak{R} \cup\{-\infty\}\}^{n}$, up $\in\{\mathfrak{R} \cup\{\infty\}\}^{n}$, and $x \in \mathfrak{R}^{n}, \mid l w<x<u p$, being $l w$ and up the lower and upper boundaries defined according to the curve under consideration.

Based on this procedure, Figure 8 shows eight examples of $\kappa-\mu$ probability density function in comparison to the empirical 

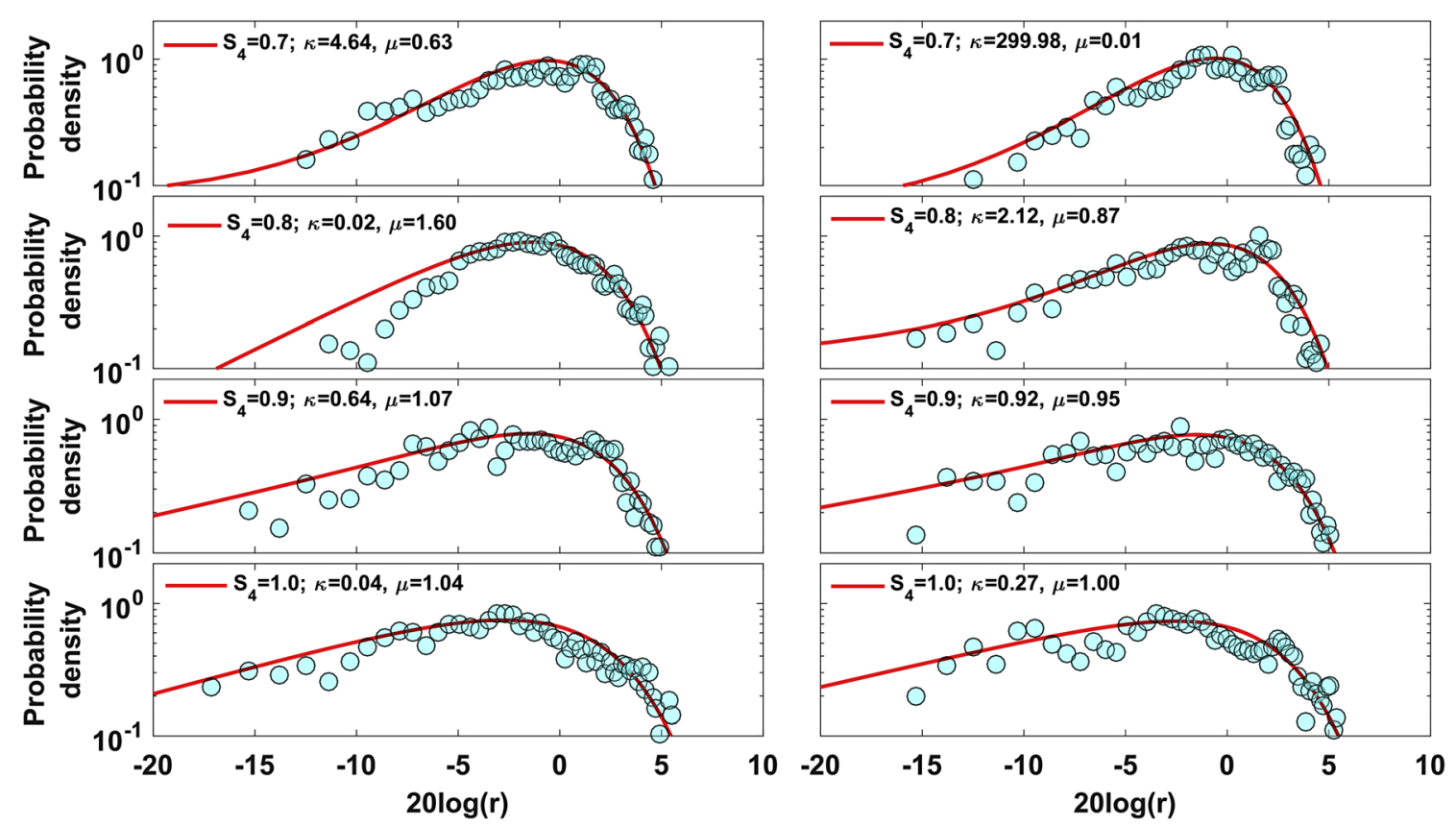

Fig. 8. Examples of empirical histograms versus the probability density function based on the $\kappa-\mu$ estimated values.

distribution (light blue circles), which are the histogram values of the normalized received signals. Scintillation data was categorized by the $S_{4}$ index from 0.7 up to 1.0 . It is possible to observe that such approach is effective in the adjustment of theoretical model to the field data and the fading coefficients were successfully estimated for all cases. The next section will discuss the fading coefficients in details.

\section{Evaluation of the statistical models performance}

In this section a comparison of the performance of the $\kappa-\mu$ model from equation (9) with the models described in Section 3, equations (2), (4) and (6), is presented. The scintillation dataset used is the same evaluated in Moraes et al. (2012) to investigate $\alpha-\mu$, Rice and Nakagami- $m$ distributions. The dataset contains approximately $60 \mathrm{~h}$ of scintillation records, in which only information collected with elevation angle greater than $30^{\circ}$ and $S_{4}$ varying from $0.4 \pm 0.025$ up to $1.0 \pm 0.025$ were considered.

The criteria adopted for the comparison of models fit accuracy was the chi-square goodness-of-fit test as given by Fontán \& Espiñeira (2008):

$$
\chi^{2}=\sum_{i=1}^{k} \frac{\left(N_{i}-n_{i}\right)^{2}}{n_{i}}
$$

where, $k$ is the total number of histogram bins selected to classify the observations, $N_{i}$ is the number of occurrences within bin $i$, and $n_{i}$ is the expected outcome inside an interval whose size depends on each distribution model to be tested. The number of DOF is $k-1$, and based on the previous works of Fremouw et al. (1978), Humphreys et al. (2009) and Moraes et al. (2012) it was chosen as DOF $=7$. Additionally, the width of the

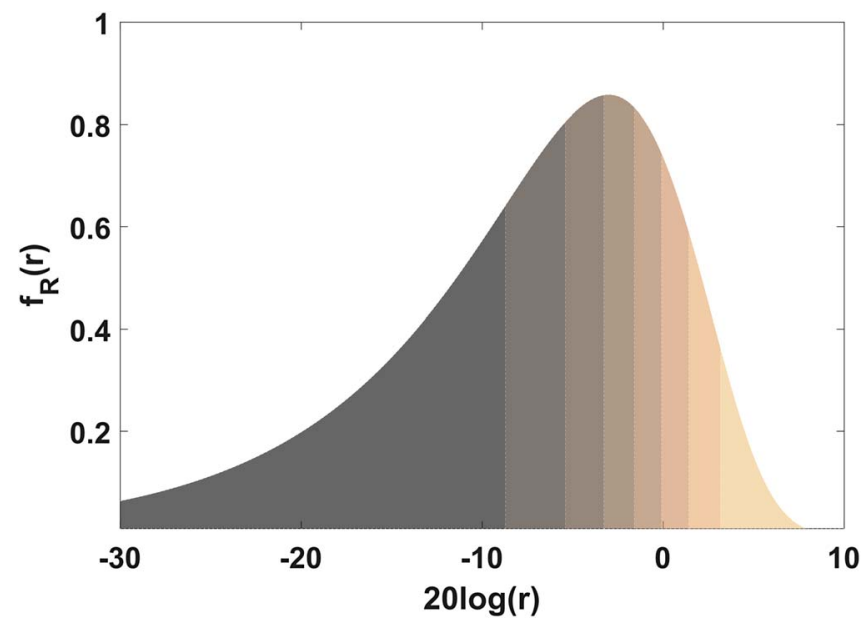

Fig. 9. Example of equally probable partition of probability density function used in the chi-square test.

bins in the histogram is varied so they became equally probable. Figure 9 shows an example of equally probable partition of the distribution for $S_{4}=1.0$, where Nakagami- $m$ and Rice models are equivalent. This is also the particular case where $\alpha=2$ in the $\alpha-\mu$ model and $\kappa=0$ in the $\kappa-\mu$ distribution. As shown in Press et al. (1992), the model whose $E\left[\chi^{2}\right]$ approximates to the DOF value is the one with the best fit performance.

In order to guarantee that the samples in the chi-square fit test were statistically independent, the scintillation data was down-sampled to twice of the decorrelation time $\tau_{0}$. The decorrelation time is an important index in scintillation analysis, and for more details see Carrano \& Groves (2010), Moraes et al. (2011) and Carrano et al. (2012). The value of $\tau_{0}$ is computed 
Table 2. $E\left[\chi^{2}\right]$ and $\sigma\left[\chi^{2}\right]$ results as functions of $S_{4}$ for different models.

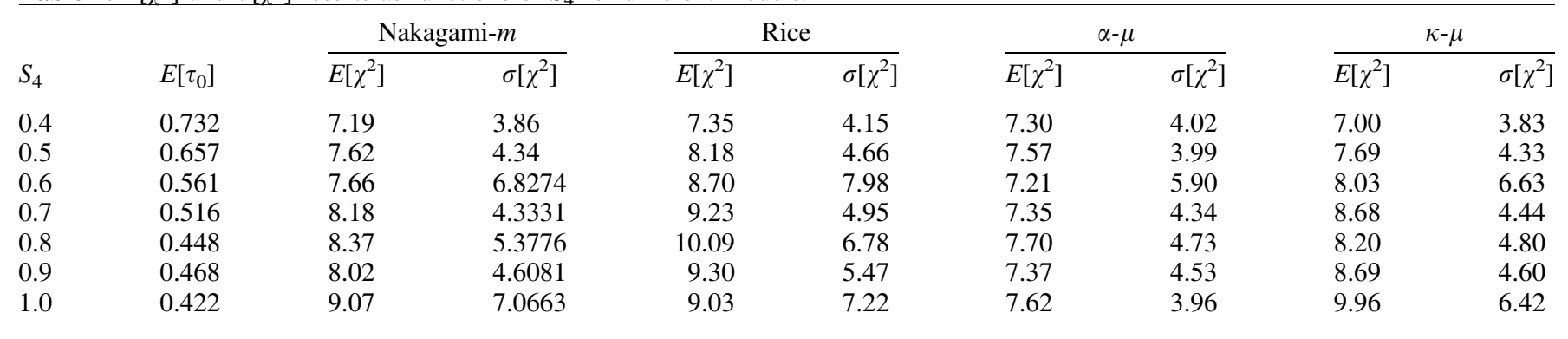

from the normalized signal $R$, by the computation of autocorrelation coefficients, given by:

$$
A_{R}(\tau)=\frac{E[(R(n)-z)(R(n+\tau)-z)]}{\sigma_{R}^{2}}
$$

where $z$ and $\sigma_{R}{ }^{2}$ are, respectively, the mean and variance of the envelope $R$. The $\tau_{0}$ is defined as the time lag at which the autocorrelation function reaches the proportion:

$$
\frac{A_{R}\left(\tau_{0}\right)}{A_{R}(0)}=\exp (-1)
$$

After the down-sampling process, only cases where each bin was expected to have 56 scintillation observations, or more, were considered for the $\chi^{2}$ test, i.e., the length of the scintillation records must be at least of 448 samples. For more details about the aforementioned procedure see Moraes et al. (2012). This was chosen based on the requirement of at least seven samples for each one of the eight bins of the equiprobable histogram used in the $\chi^{2}$ test. The average values of the decorrelation time $E\left[\tau_{0}\right]$ are shown in the second column of Table 2 .

The results of the chi-square test for the evaluated theoretical models are shown in Table 2. The steps below summarize the procedure for $\chi^{2}$ computation:

(i) Scintillation data was down-sampled to twice of the decorrelation time $\left(\tau_{0}\right)$;

(ii) The equally probable histogram intervals (bins) for the four candidate distributions were computed;

(iii) The $\chi^{2}$ was computed based on equation (13);

(iv) The mean value, $E\left[\chi^{2}\right]$, of the $\chi^{2}$ results, for each $S_{4}$ range, was computed as well as its standard deviation, $\sigma\left[\chi^{2}\right]$.

The results presented in the Table 2 confirm that the $\alpha-\mu$ model presents slight better result in general when compared with the other models for the whole range of $S_{4}$. This can be verified by $E\left[\chi^{2}\right]$ which is the parameter that describes the capability of the model to fit the received signal. The $E\left[\chi^{2}\right]$ values for the Nakagami- $m$ and $\kappa-\mu$ models are very close, presenting a similar fit performance. Finally, the Rice model presents reasonable results, but it has the worst performance among the models tested in terms of fit capability.

The fact that models with one more DOF are expected to perform better was already discussed as showed in Moraes et al. (2012). While Nakagami- $m$ and Rice distributions correspond to single curves, the $\kappa-\mu$ and $\alpha-\mu$ models have infinite shapes as long they satisfy, respectively, equations (8) and (12). In order to better understand the fit results achieved, Table 3 presents the $E[\alpha]$ and $E[\kappa]$ results as functions of $S_{4}$ for the $\alpha-\mu$ and $\kappa-\mu$ models for the cases listed in Table 1 . It worth mentioning again that, for the same $S_{4}$ value, as $\alpha$ or $\kappa$ increases, more severe is the scintillation scenario and the tails of the distributions tend to rise as these coefficients increase.

Revisiting Figure 7 , it is possible to verify that $\kappa-\mu$ model has a more elevated tail than $\alpha-\mu$ distribution (Fig. 5). For example, analyzing all cases in Figure 7 where $\kappa=0$ and comparing to the respective cases for the $\alpha-\mu$ model, it is possible to verify that in Figure 5 the $\alpha-\mu$ domain of the curves covers a region of values where $\kappa-\mu$ model does no reach. Taking the examples of the left panel of Figure 8 , where $S_{4}=0.8$, $\kappa=0.02$ and $S_{4}=0.9, \kappa=0.64$ it is possible to note that the empirical probabilities are below the theoretical $f_{R}(r)$ in the tail region. These examples show another restriction of this model $(\kappa-\mu)$ for cases where the fading distributions presents narrower profiles with more concentrated fading events.

Extending the discussion between the models, for comparison merit, Figure 10 presents the curves of the models described by equations (2), (4), (6), and (9), respectively. For the $\alpha-\mu$ and $\kappa-\mu$ models the values adopted are based on the average values from Table 3 . Analyzing Figure 10, it is possible to observe that $\kappa-\mu$ is always closer to Rice model while $\alpha-\mu$ curves are always under the Nakagami- $m$ in a sense of wideness, except in the case where $S_{4}=1.0$, when Nakagami- $m$ and Rice have equivalent curves. Also, the $\kappa-\mu$ distribution has the especial feature to becomes Rice when $\kappa=1$ and $\mu=1$ under this condition. Even in this case it is clear that the $\alpha-\mu$ curve describes a scenario with more concentrated occurrence of values. Indeed, that is the reason why $\alpha-\mu$ model fits better the analyzed dataset. The $\alpha-\mu$ curves are in a region not covered by the $\kappa-\mu$ model as discussed. This is more evident for $S_{4} \geq 0.9$, where the $E[\kappa] \rightarrow 0$ which shows a tendency of more concentrated profiles. Hence, the $\kappa-\mu$ model may be considered a conservative model when compared to the empirical probability density which is narrower.

These results presented in Table 3 are consistent with Moraes et al. (2018b) where the $E[\alpha]$ values are presented for the same location besides three others in Brazilian territory and the average value for $S_{4} \geq 0.7$ is $\sim E[\alpha]=2$ but with significant concentration of occurrences in the region where $\alpha<2$. This fact justifies the better fit performance of $\alpha-\mu$ model in comparison to the $\kappa-\mu$. 

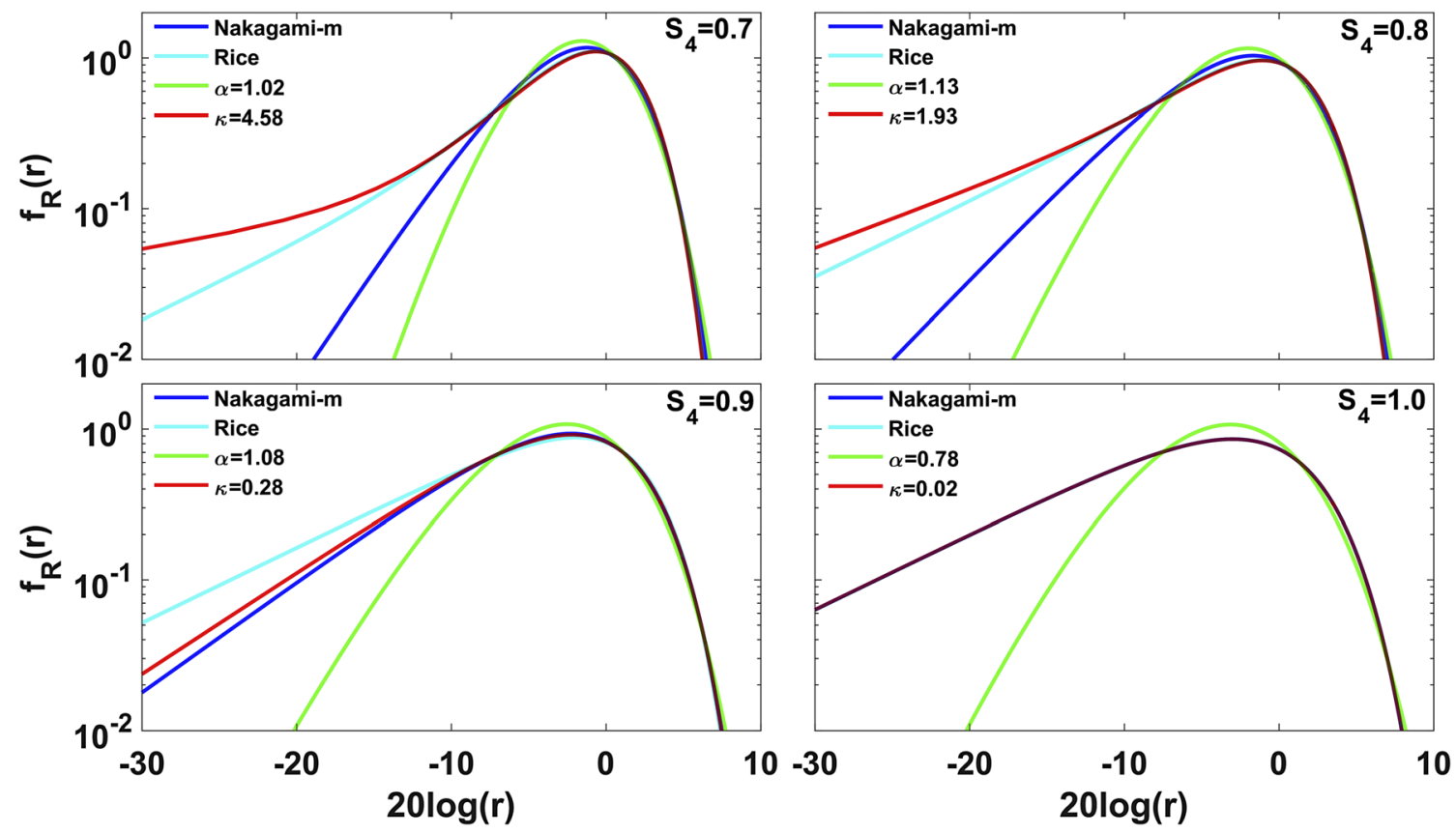

Fig. 10. Comparison between $\alpha-\mu$ and $\kappa-\mu$ models.

Table 3. $E[\alpha]$ and $E[\kappa]$ results as functions of $S_{4}$ for the tested $\alpha-\mu$ and $\kappa-\mu$ models.

\begin{tabular}{lrllll}
\hline$S_{4}( \pm 0.025)$ & 0.6 & 0.7 & 0.8 & 0.9 & 1.0 \\
$E[\kappa]$ & 103.27 & 4.58 & 1.93 & 0.28 & 0.02 \\
$E[\alpha]$ & 0.97 & 1.02 & 1.13 & 1.08 & 0.78 \\
\hline
\end{tabular}

\section{Summary and conclusions}

In the ionosphere, at low-latitude regions, the occurrence of plasma bubbles usually affects satellite communications, in particular GNSS users. The plasma density irregularities result in amplitude scintillation for the signals that cross these irregular structures. Over the Brazilian sector, the geomagnetic field configuration presents a large magnetic declination angle which aligns with the solar terminator during the summer, such configuration is a propitious scenario to catalyze the generation of these irregularities, and thus, longitudinal asymmetries in the phenomenology are verified. The presence of these plasma structures results in an increasing in the availability issues. Hence, it is important to develop models and methods to describe the effects of scintillation. In this work, an evaluation of statistical models was done to show how accurate they are in the characterization of amplitude scintillation.

Previously, in works like Fremouw et al. (1978, 1980) and Humphreys et al. (2009), the statistical analysis of amplitude ionospheric scintillation was performed using single parameter distributions such as Rice or Nakagami- $m$. More recently in Moraes et al. (2012) the authors proposed the use of a two parameter based distribution for this application, namely the $\alpha-\mu$ model. The results of this work corroborated the intuitive hypothesis that the usage of a distribution with an additional parameter could possibly fit the empirical data more adequately.
However, this claim needs to be checked using other non-single parameter distributions. Within this context, the $\kappa-\mu$ model is a viable candidate once according to Yacoub (2007b) this model was suited for line-of-sight applications as in satellite communication scenario. The present work showed the flexibility of bivariate distributions like the $\alpha-\mu$ and $\kappa-\mu$ models in comparison with the single parameter distributions Nakagami- $m$ and Rice. Also, using the $\chi^{2}$ test, the four models, Nakagami- $m$, Rice, $\alpha-\mu$ and $\kappa-\mu$ were tested. The results demonstrated that the $\kappa-\mu$ and Nakagami- $m$ models present better performances than the Rice distribution to describe the statistics of amplitudes during scintillation events for all $S_{4}$ range evaluated in this analysis. The $\chi^{2}$ outcomes evidenced that the $\alpha-\mu$ distribution, however, outperformed all the distributions, reaching the best results in the fitting tests.

For the $\kappa-\mu$ model, the limitations of the moment-based estimator for the fading coefficients of scintillation data were shown and one alternative approach using weighted least square fit method was proposed and tested.

The analysis performed in this work still showed in detail the influence of the fading coefficients $\alpha$ and $\kappa$ in the shape of its probability density functions. For both models, as $\alpha$ or $\kappa$ increases, more severe are the fading events considering the same $S_{4}$. This, for example, may help to justify why equal values of $S_{4}$ may have different impacts on the receiver. In this sense, $\alpha$ and $\kappa$ may be complementary parameters that synthesize the severity of the fades as discussed in Moraes et al. (2018a). This is an important aspect that may be useful for positioning algorithms like in Aquino et al. (2009) or to estimate the receiver errors like in Aquino et al. (2007).

Another aspect discussed in this work was the difference between the proposed models, especially $\alpha-\mu$ and $\kappa-\mu$. It has been shown that the $\kappa-\mu$ distribution has a more raised tail feature. The least conservative case of this model occurs when $\kappa=0$. Meanwhile, the $\alpha-\mu$ model has a broader coverage, acting 
in an area where the $\kappa-\mu$ model can not reach and yet being able to generate shapes in the region where $\kappa-\mu$ operates. This behavior justifies the $\chi^{2}$ results. Together with this analysis, the mean values of $\alpha$ and $\kappa$ were introduced. These fading coefficients values may serve for future theoretical work using these models.

Finally, this work has shown that bivariate distributive models may help to describe scintillation statistics in a wider domain. The results of the fit tests showed that the $\kappa-\mu$ model makes a fair adjustment to the empirical curves. The usage of the $\kappa-\mu$ model may be then indicated for applications when more cautious approaches are needed, such as aircraft positioning and navigation systems due to its flexibility. Although this distribution is not the one that best describes the scintillation statistics due to its feature of a relatively more raised tail. This model may be particularly interesting option for analysis of channels, for example, that involve scintillation with other phenomena like, for instance, multipath.

Acknowledgements. A.O. Moraes would like to thank the support from Conselho Nacional de Desenvolvimento Científico e Tecnológico (CNPq) through Award 314043/2018-7. E. R. de Paula is supported by CNPq award number (PQ) 310802/2015-6. J. Sousasantos is gratefull to CAPES and Fundação de Amparo à Pesquisa do Estado de São Paulo (FAPESP) support under grant 2018/06158-9. B.C. Vani thanks to INCT GNSS-NavAer, funded by $\mathrm{CNPq}$ process 465648/2014-2 and FAPESP 2017/01550-0. The authors are grateful to Dr. Mangalathayil Abdu, Prof. Waldecir Perrella and Prof. Michel Yacoub for their comments and suggestions which have significantly improved this work. The editor thanks two anonymous referees for their assistance in evaluating this paper.

\section{References}

Abdu MA, Batista IS, Sobral JHA. 1992. A new aspect of magnetic declination control on equatorial spread-F and $\mathrm{F}$ region dynamo. $J$ Geophys Res 97(A10): 14897-14904.

Aquino M, Andreotti M, Dodson A, Strangeways H. 2007. Improving the GNSS positioning stochastic model in the presence of ionospheric scintillation. Adv Space Res 40(3): 426-435.

Aquino M, Monico J, Dodson A, Marques H, De Franceschi G, Alfonsi L, Romano V, Andreotti M. 2009. Improving the GNSS positioning stochastic model in the presence of ionospheric scintillation. J Geodesy 83: 953-966. DOI: 10.1007/s00190-009-0313-6.

Banerjee PK, Dabas RS, Reddy BM. 1992. C and L band transionospheric scintillation experiment: Some results for applications to satellite radio systems. Radio Sci 27: 955-969. DOI: 10.1029/92RS01307.

Beach TL, Kintner PM. 2001. Development and use of a GPS ionospheric scintillation monitor. IEEE Trans Geosci Remote Sens 39: 918-928.

Carrano CS, Groves KM. 2010. Temporal decorrelation of gps satellite signals due to multiple scattering from ionospheric irregularities. Proc. of the 2010 Institute of Navigation ION GNSS meeting Navigation ION GNSS meeting.

Carrano CS, Valladares CE, Groves KM. 2012. Latitudinal and local time variation of ionospheric turbulence parameters during the Conjugate Point Equatorial Experiment in Brazil. Int $J$ Geophys, 2012: 103963. DOI: $10.1155 / 2012 / 103963$.
Coleman TF, Li Y. 1994. On the convergence of interior-reflective Newton methods for nonlinear minimization subject to bounds. Math Program 67: 189-224. DOI: 10.1007/BF01582221.

Coleman TF, Li Y. 1996. An interior, trust region approach for nonlinear minimization subject to bounds. SIAM J Optim 6: 418-445.

Datta-Barua S, Lee J, Pullen S, Luo M, Ene A, Qiu D, Zhang G, Enge P. 2010. Ionospheric threat parameterization for local area global-positioning-system-based aircraft landing system. J Aircraft 47: 1141-1151. DOI: $10.2514 / 1.46719$.

Fremouw EJ, Leadabrand RL, Livingston RC, Cousins MD, Rino CL, Fair BC, Long RA. 1978. Early results from the DNA wideband satellite experiment - Complex-signal scintillation. Radio Sci 13: 167-187.

Fremouw EJ, Livingston RC, Miller DA. 1980. On the statistics of scintillating signals. J Atmos Terr Phys 42: 717-731.

Fontán FP, Espiñeira PM. 2008. Modelling the wireless propagation channel: a simulation approach with Matlab. Wiley. https:// onlinelibrary.wiley.com/doi/book/10.1002/9780470751749.

Guo K, Zhao Y, Liu Y, Wang JL, Zhang CX. 2017. Study of ionospheric scintillation characteristics in australia with GNSS during 2011-2015. Adv Space Res 59: 2909-2922.

Humphreys TE, Psiaki ML, Hinks JC, Kintner PM Jr. 2009. Simulating ionosphere-induced scintillation for testing GPS receiver phase tracking loops. IEEE J Sel Top Signal Process 3: 707-715.

Klobuchar JA. 1987. Ionospheric time-delay algorithm for singlefrequency GPS users. IEEE Trans Aerospace Electron Syst 3: $325-331$.

Marques HA, Marques HAS, Aquino M, Veettil SV, Monico JFG. 2018. Accuracy assessment of precise point positioning with multiconstellation GNSS data under ionospheric scintillation effects. J Space Weather Space Clim 8: A15. DOI: 10.1051/swsc/2017043.

Moraes AO, Rodrigues FS, Perrella WJ, Paula ER. 2011. Analysis of the characteristics of low-latitude GPS amplitude scintillation measured during solar maximum conditions and implications for receiver performance. Surv Geophys 33(5): 1107-1131. DOI: $10.1007 / \mathrm{s} 10712-011-9161-\mathrm{z}$.

Moraes AO, Paula ER, Perrella WJ, Rodrigues FS. 2012. On the distribution of GPS signal amplitudes during the low-latitude ionospheric scintillation. GPS Solut 17(4): 499-510. DOI: 10.1007/ s10291-012-0295-3.

Moraes AO, de Paula ER, Muella MTAH, Perrella WJ. 2014. On the second order statistics for GPS ionospheric scintillation modeling. Radio Sci 49(2): 94-105. DOI: 10.1002/2013RS005270.

Moraes AO, de Muella MTAH, Paula ER, de Oliveira CBA, Terra WP, Perrella WJ, Meinbach-Rosa PRP. 2017. Statistical evaluation of GLONASS amplitude scintillation over low latitudes in the Brazilian territory. Adv Space Res 61(7): 1776-1789. DOI: 10.1016/j.asr.2017.09.032.

Moraes AO, Vani BC, Costa E, Abdu MA, de Paula ER, et al. 2018a. GPS availability and positioning issues when the signal paths are aligned with ionospheric plasma bubbles. GPS Solut 22: 95. DOI: 10.1007/s10291-018-0760-8.

Moraes ADO, Vani BC, Costa E, Sousasantos J, Abdu MA, et al. 2018b. Ionospheric scintillation fading coefficients for the GPS L1, L2, and L5 frequencies. Radio Sci 53: 1165-1174. DOI: 10.1029/2018RS006653.

Press WH, Teukolsky SA, Vetterling WT, Flannery BP. 1992. Numerical recipes in $C$ : The art of scientific computing, Second edition. Cambridge University Press, Cambridge, UK. Chapter 15.

Seo J, Walter T, Enge P. 2011. Availability impact on GPS aviation due to strong ionospheric scintillation. IEEE Trans Aerospace Electron Syst 47(3): 1963-1973. DOI: 10.1109/TAES.2011. 5937276. 
Simon MK, Alouini M. 2006. Digital communications over fading channels. Wiley, New York, NY.

Sobral JHA, Abdu MA, Takahashi H, de Taylor MJ, Paula ER, Zamlutti CJ, Aquino MG, Borba GL. 2002. Ionospheric plasma bubble climatology over Brazil based on 22 years (1977-1998) of $630 \mathrm{~nm}$ airglow observations. J Atmos Sol-Terr Phys 64: 1517-1524.

Sousasantos J, de Oliveira Moraes A, Sobral JHA, Muella MTAH, de Paula ER, Paolini RS. 2018. Climatology of the scintillation onset over southern Brazil. Ann Geophys 36: 565-576. DOI: 10.5194/angeo-36-565-2018.

Stolle C, Lühr H, Rother M, Balasis G. 2006. Magnetic signatures of equatorial spread $\mathrm{F}$ as observed by the CHAMP satellite. J Geophys Res 111: A02304. DOI: 10.1029/2005JA011184.

Thébault E, Finlay CC, Beggan CD, Alken P., Aubert J. et al. 2015. International geomagnetic reference field: the 12th generation. Earth Planet Space 67: 79. DOI: 10.1186/s40623-015-0228-9.
Veettil SV, Aquino M, Spogli L, Cesaroni C. 2018. A statistical approach to estimate Global Navigation Satellite Systems (GNSS) receiver signal tracking performance in the presence of ionospheric scintillation. J Space Weather Space Clim 8: A51. DOI: 10.1051/ swsc/2018037.

Xiong C, Stolle C, Lühr H. 2016. The Swarm satellite loss of GPS signal and its relation to ionospheric plasma irregularities. Space Weather 14: 563-577. DOI: 10.1002/2016SW001439.

Yacoub MD. 2007a. The $\alpha-\mu$ distribution: A physical fading model for the Stacy distribution. IEEE Trans Vehic Technol 56: 27-34. DOI: 10.1109/TVT.2006.883753.

Yacoub MD. 2007b. The $\kappa-\mu$ distribution and the $\eta-\mu$ distribution. IEEE Antennas Prop Mag 49: 68-81.

Yeh KC, Liu CH. 1982. Radio wave scintillation in the ionosphere. Proc of the IEEE 70(4): 324-360.

Cite this article as: Moraes A, Sousasantos J, de Paula ER, da Cunha J, Lima Filho VC, et al. 2019. Performance analysis of $\kappa-\mu$ distribution for Global Positioning System (GPS) L1 frequency-related ionospheric fading channels. J. Space Weather Space Clim. 9, A15. 